\title{
CULTIVO DE CIANOBACTERIAS DE COSTRAS ALGAL-LIQUÉNICAS DE LAS LOMAS DE PACHACAMAC, LIMA
}

\author{
HAYDÉ MavToxa $T^{2.2}$ \\ Jose Gounz C \\ Dino Maniva $C^{3}$ \\ Gonuvin Vene ${ }^{2}$
}



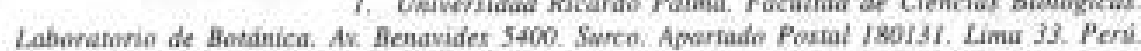 \\ 2. Muses de Histaria Nanrol \\ Labovatario Simbiasis Vegetal. UNMSM An. Avenaies 1256. Apertado Postal 140434 Lima IS. Perif; \\ 3. Universidad Naciomal de Thujillo, Le Libertad Peri
}

\begin{abstract}
RESUMEN
Las comunidades crustíceas de suclos en las ecosistemas destrticos del muado tienen una microflora peculiar de importancia en la formacice y estabilizacica de los suelos. El desierno Peruane Central se caracteriza por la formación de lomas y costras microbióticas dehido a la hamedad. Colecciones de tabos de las costras desérticas de las lomas de Pachacanac (Jatua Sisa) se realizo en noviembre de 1995 y 1997. Ineculaciones de las costras y cultives en modios Basal de Bold y N8 se realizaron a temperaturas promedio de $21^{\circ} \mathrm{C}$ y fuerou seguidas basta 1.5 anos. La colonizacióe algal-liguetnica de las costras por las cianobacterias Scytoeema erispam, Mieroceleus vaginatus y Nestec commune (Scytonemataceac, Oscillatoriaceae y Nostocaceae) y los tiquenes Parmelia (Parmeliaceae) y Ramalina (Ustueaceae) carkikterizos a las comunidades saxicolas. Los tales cianohacaeriales tavieron mejor desarrollo en el periodo himedo (noviembre, 97) y en los cultivos. La variabilidad moxfotogiea de les ulos cianohacteriales con el desarnollo de la pared y estuche extracelulares en porciones de talos latentes asi come de estadios de resisteacia (bormocistos) se presenof en periodos secos (noviemhre, 95). La formacibes

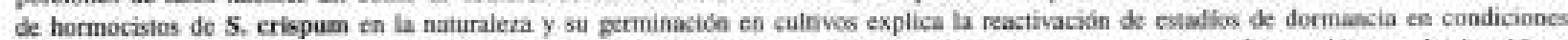
hüraedas. Además la capacidad diazolrufica de las especies heterocistadas S. erispam y N, eommane favoreció su colonización y perenaización on estos eossistemas. La plasticidad fenotipica de los talos ciancbacteriales en coodiciones climaiticas fluctaastes en las lomas detrostraron su estrategia de tokrancia al estrés hidrico por desecacióto.
\end{abstract}

\section{SUMMARY}

CYNOBACTERIAL CULTURES OF ALGALLICHFN CRUSTS FROM THE LOMAS DE PACHACAMAC, LIMA Soil crust comen:nities in desert ecosystems have pecaliar micmolora of relevance in soil formation and stabrilization. Central Peruvian desert has the lamas fornatioss due to the moisture period which allows the development of microbintic crusts. Collections of desert crusts were done at Pachacamac lomas $\left(12^{2} 13^{\circ} \mathrm{LS}\right)$ in noventer 95, 97. Crust inoculations in culture modia (Basal Bobs. N8) at about $21^{2} \mathrm{C}$. were followed up to 1.5 year. Algal-lichen crust colonization was formed by the cyasobacteria Segtoeena crispum, Microeoleus vaginatus and Nestoc commune (Scyloncmatactae, Oscillasoriacese and Nostocactac) and the lichens. Parmelia (Parmeliaceac) and Ramalina (Usnesceae) which typified the saxicolous communities. Morphological variability of cyanobacterial thaili with the well-developed cell walls and extracel Jular sheaths in laten thalli and resistant stages as harmocysts were notocious in dry periods inovember 95). Cyanobacterial species had betier growth during the maistare period af The Nifo Phenomenam (november 97) and in cultures. Nature formation of $\mathbf{8}$. erispum hormecysts and their germination in cultures explain the reactivation of domaincy stages under moisturv coesiticas. Besides, the diazodrophic cipacity of $\mathrm{S}$. crispem, beterocystous species, as well as $\mathrm{N}$. commune farours their colonization and perennization in the desert. Therefore. phenotypic plasticity of cyanobacterial thalli under fluctuating elimasie coeditions in the lomas evidenced their tolerance strakegies under stress desiocation. Algal morphological variability and esological adaptatices in saxicoloes coenmunities coniplemented with cultures let ins understanding the al gat-lichen colonization of tropical desent ecosystems.

\section{INTRODUCCIÓN}

El territorio peruano costero comprende una variedad de ecosistemas áridos y semiáridos tropicales entre los cuales se presentan peculiares formaciones vegetales como las lomas favorecidas por la humedad de los frecuentes estratos nebulosos.

Los talos algal-liquénicos colonizan una variedad de hábitats que incluyen a los más extremos como los desiertos que son excluyentes para otros organismos fotótrofos. Las costras desérticas (microbióticas, eriptogámicas o cianobacteriales) constituyen microhábitats terrestres estables con microambientes peculiares con gradientes fisico-quimicas que ejercen resistencia a las fuerzas erosionales (BELNAP \& GARDNER 1993, GARCIA-PICHEL \& BELNAP 1996).

Las cianobacterias constituyen componentes dominantes de las comunidades fotosintéticas terrestres (suelos, rocas) de regiones áridas y semiáridas, frias o calientes siendo la cianobacteria colonial Nostoc commune considerada como una de los más efectivas sobrevivientes 
de nuestro ecosistema (FOREST \& WESTON 1966, FRIEDMANN \& GALUN 1974. FRIEDMANN 1980 , WHITTON 1987), Inicialmente la estabilidad de las cortras superficiales terrestres de regiones áridas y semiáridas estuvo asociada con la presencia de algas y en determinadas áreas (Planicie de Colorado, USA) las eoctras algal-fiquénicas constituyen hasta un $70 \%$ de la comunidad biótica con la predominancia de la cianobacteria Milcrocoleus vaginatus (SHIE] Det al, 1957, ANDERSON \& RUSHFORTH 1976, BELNAP 1990). Las costras cianobacteriales modifican la microestructura de kis suelos estimulando su estabilidad con la consecuente reducción en la erosión del suelo por aire y agua, favoreciendo la retención de humedad e incrementando su fertilidad (SHIEI DS \& DURELL 1964, CAMPBEH 1979, BEL NAP ¿ GARDNER 1993).

Cianobocterias del desierto de Atacama, norte de Chale, fueron obtenidas en cultivo regisiraindose las especies Schizothrix calcicola (Agardh) Gomont, Anacystis montana (Lightf.) Drouct \& Daily y Coccochloris peniocystis Drouet \& Daily (FOREST \& WESTON 1966). En nuestro país, tres especies de cianobacterias han sido reportadas en los suelos de alrededores de Lima: M. paludosus, Nodularia spumigena y Cylindrospermum licheniforme (ZÚNICAA 1989).

Debido a la escasa información respecto a las costras algal-liquémicas terrestres en nuestra costa desćrtica y a sus especiex representativas de gran significado ecologico, el presente trabajo (primera parte) presenta algunas estrategias de sobrevivencia de las cianobacterias de las lomas de Pachacamac en poblaciones naturales y de cultivo.

\section{MATERIAL YMÉTODOS}

Las lomas de Pachacumac están localizadas a $42.5 \mathrm{~km}$. al sur de Lima, aproximadameate a $12^{\circ} 13^{\prime}$ LS y 76"51' LO Colecciones de costras desérticas fueron realizadas siguiendo la metodologia de colección estándar con el raspado de superficies rocosas y del suelo en las lomas de Pachacamac (Jatun Sisa) en noviembre 1,95 y noviembre 30. 97. Inoculaciones de los talos crustáceos algalliquénicos fueron realizados en los medios inergánicos de cultivo Basal de Bold (BISCHOFF \& BOL.D, 1963) de pH 6.5 - 6.8 y N8 (Instituto del Carbón Biológico de Dormund) de $\mathrm{pH} 6.1$ - 6.4. Técnicas de aislamiento y subcultivos de las microalgas se realizaron a temperatura promedio de $21^{\circ} \mathrm{C}$. La iluminación fue proporcionada por lámparas fluorescentes blancas de $40 \mathrm{~W}$ así como iluminación natural a través de una ventana. Observaciones microscópicas de los talos en medios de cultivos líquido y agarizado (1.8\%) fueron seguidos hasta los 1.5 años. La identificsción de especies fue realizada mediante bibliografía especializada (GEITLER 1932, KOMAREK \& ANAGNOSTIDIS 1989)

\section{RESULTADOS}

\section{Área de Estudio}

Las condiciones climáticas de la Costa Peruana Central ocasionan un clima moderado, con precipitación muy escasa e irregular y formación regular de estratos neblinosos principalmente en inviemo y que aún en verano es posible reconocerlos ocasionalmente. Durante las dos coleccioses realizadas en primavera tuvo contrastes climátioos notorios, En la primera colección las condiciones ambientales fueron más bien secas comparadas con la intensa neblina que cubria las lomas aunadas con garúas intensas e irregulares durante la segunda colección que correspondi6 a los evenios climáticos durante el Fenómeno de el Niño 1997 .

Las costras desérticas bien distribuidas en las lomas están sujetas a los contrastes climáticos (sequedadhumedad) y a las modificaciones del ecosistema causadas por la influencia del ganado (vacuno, ovino y caprino), que pastorea contribuyendo a la desertificación que se acentúa durante la estación seca (verano).

\section{Comanidades algai-liquuénicas desérticas}

Las cosiras algal-liquénicas en su mayoria saxicolas, grisdiceas, pardo oscuras a negruzeas, se presentaron aplanadas de superficie áspera y ondulada, fácilmente quebradizas y pulverulentas por la desecación a la cual estuvieron expuestas sobre el sustrato rocoso con heterogeneidad espacial como la superficie irregular a mediados de primavera (noviembre 1,95 ) en las Jornas de Jatun Sisa, Pachacamac. Esta compleja colonización criptogámica de las costras desérticas desecadas y distribuidas principalmente en parches estuvo compuesta por cianobacterias generalmente asociadas con talos liquénicos foliosos como Parmelia (Parmeliaceae)(Fig.1). La estructura algal en su mayoria en estado anabiótico (metabolismo reducido), por disminación del contenido de agua (humedad) estuvo dominada por los talos filamentosos de las cianobacterias Scytonema crispum (C.A. Agardh) Bornet (Scytonematacene), Microcoleus vaginatus Gomont (Oscillatoriaceac) junto con las formas coloniales mucilaginosas de Nostoc commune Vaucher. Las formas filamentosas entrelazadas (red) y algo

Fig. 1. Cemmaidades alpal-liquenicas de cootras descrticas sacicolas. Fig.2, Pilamento compuesto de Microcoleus vaginatus de comonidad saxicola con particulas de spelo adheridas a estuche mocilacinose Figs, 3-4, Flarestos de Scytonema crispoum de cormunidad szuicala. Fig. 3. Hormocistos ierminales formados en ramas falsas pares rodeados de estuche essratificado. Fig. 4. Ramificasiole falsa con heterocistos en so anigen. Figs.5-7, Cultivo liquado de S. crispam en medio Basal Bold de inosulo inicial de 1 semana. Pig. 5. Germinación de barmocisto en erapa isicial con hinchamieato de estuche. Fig.6. Activacióa de célalas vegetativas latebies de hormocisto. Fig.7. Emerefencia de células vegerativas apicales del hormocisto. Eiscala en Fig 2 villida para Figs.2-7 

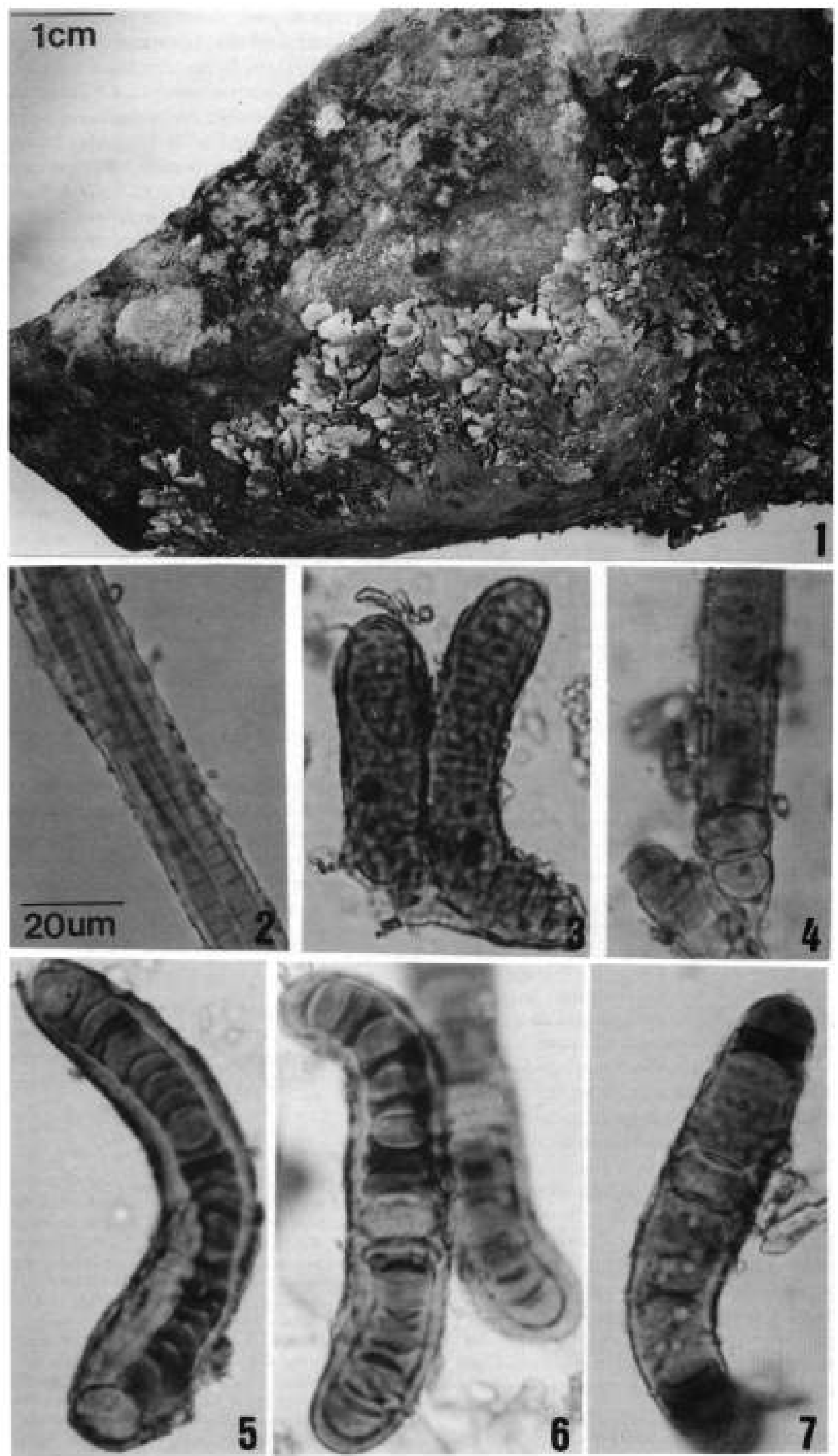
enrostadas de las cianobacterias, con células viables contraidas casi en forma uniforme, exhiben la adhesión de partículas del suelo en la superficie de sus estuches extracelulares mucilaginosos además de sostener particulas en la red de los filameatos que produce la agregación de las partículas del suelo durante la colonización algal-liquénica.

Las costras desérticas fueron humedecidas a mediados de primavera (noviembre 30,97 ) por las inusuales. garúas algo persistentes durante el fenómeno de El Niño. Los estuches extracelulares de los filamentos secos. sinuosos y enruscados por la sequedad se hinchan en perícodos húmedos cubriendo los suelos superficiales. La turgencia de las colonias terrestres y saxícolas de Nostoc commune, de coloración verde oscura, verde pardusca o verde negruzca permitieron su reconocimiento y distribuciôn bastante extendida en las lomas en estaépoca. En las superficies rocosas, las colonias de $\mathbf{N}$. commune estuvicron asociadas con las matas pardo oscuras de $\mathbf{S}$. crispum, de aspecto cespitoso por la densidad de los filamentos, que a su vez estuvieron asociados con el liquen fruticoso Ramalina (Usneaceae) y en menor proporción de musgos higrofiticos. Las costras rugosas humedecidas de N. commune mostraron proliferación de colonias que emergen de los talos crustáceos basales. La parte basal crustácea y la parte erguida con colonias globulares permitió relacionarlos con salos diferenciados como los beterotricos.

\section{Caracrentación de poblaciones de Scytonema crispum}

Durante el período seco, la morfología compleja de las poblaciones algales de $\mathbf{S}$. crispum somo las matas negruzcas o pardo oscuras, cespitosas o con apariencia de motas presentaron filamentos largos, curvados, ligeramente atenuados y ramificados, de 15 a 18 um de diametro. Los filamenios estuvieron roxieados de estuches gruesos, estratificados y pigmentados (pardo amarillento ascuro), de hasta $3.2 \mu \mathrm{m}$ de espesor y con las células escasamente visibles. La mayoría de estos talos filamentosos vegetativos resistieron periodos desfavorables con la consecuente sequedad de los talos y con el desarrollo del estuche gelatincso que demostro ta habilidad de la especie bajo condiciones de estrés por desecación. En general, a lo largo de los filamentos la mayoria de células son pardo doradas, pardo verdosas claras y otras pálidas. Las células vegetativas alcanzaron de 10,2 a $12.8 \mu \mathrm{m}$ de diámetro y de 2 a $3 \mu \mathrm{m}$ de longitud. Los heterocistos hemiesféricos u ovoides alcanzaron de 11 . 8 a 16 um de diámetro por 6.4 a 11.8 m de longitud. Hotmocistos de filamentos cortos, rodeados de estuche denso, frectuentes, con diámetro de 15 a 18 mm. Ellos se formaron por enquistamiento de hormogonios mótiles en la mayoria de los casos. Sin embargo, los hormocistos intercalares se originaron al desprenderse los homnogonios del filamento priscipal a de las ramas por fragmentación. Durante la germinación también se reconociós la presencia de beterocistos (Figs, 3-4).

Los tales parduscos de S. crispum obtenidos en periodo húmedo como ocurrio durante el fenómeno de El Ní6o (noviembre 30,97 ) presentaron células vegetativas discoidales intensamente azul verdosas en su mayoria. de 14 a $19.3 \mu \mathrm{m}$ de diámetro por 3.2 a $8.6 \mu \mathrm{m}$ de longitud. Estas células fueron mejor desarrolladas en comparación con las células vegetativas de la primera colección de periodo más bien seco (noviembre 1.95) que alcanzaron dimensiones menores $(10.2$ a $12.8 \mu \mathrm{m} \times 2$ a $3 \mu \mathrm{m})$. Igualmente los filamentos con frecuentes hormogonios alcanzaron hasta $30 \mu \mathrm{m}$ de diámetro aunque el estuche mucilaginoso pardo amarillento fue más delgado.

\section{Caracterizaciōn de poblaciones Microcoleus vaginatus}

Se reconccieron talos filamentosos de M. vaginatus sólo en época seca con filamentos complejos de 13.9 a 37.5 um de diámetro. Estos filameatos exhibieron pxeas o numerosos tricomas superpuestos formando paquetes fasciculares, rodeados de un estuche grueso común mucilaginoso con particulas de suelo adheridas (Fig. 2). Tricomas libres en los ápices de los fasciculos fueron frecuentes. Las células discoidales alcanzaron de 4.8 a 6.4 um de diámetro poe 2.1 a 3.7 un de longitud. Celula apieal de $3.2 \mu \mathrm{m}$ de diámetro por $2.7 \mu \mathrm{m}$ de longitud. Los bormogonios liberados dentro del filamento compuesto desarrollan en su interior e incrementan el numero de tricomas de los filamentos y éstos a su vez de los fasciculos.

\section{Cultivos Cianobacteriales}

La complejidad morfológica de las poblaciones algales. saxicolas fue determinante para demostrar la importancia de estos ecotipos cianobacteriales que complementades con sus cultivos evidenció el desarrollo de formas filamentosas de rápido crecimiento como Microcoleus vaginatus $y$ Scytonema crispum.

\section{Scytonema crispum}

El proceso de reactivación de las formas latentes de los talos cianobacteriales de Scytonema crispum como filamentos en dormancia y hormocistos presentes en las costras desérticas fue seguido en cultivo (Figs. 5-7).

Figs \$.9. Cultivo liquido en medio Basal Bold de inórale inicial (costrat de 2 meses. Fig. 8 . Pensehos aruf verdosos de filamentos ramificados de Scytasema crispun en parte soperior de nata pardusca de Microceleus vaginatus. Fig. 9. Filamesto de S. crispem cont ramificación falsa en pares y celulas vegetativas effericas azul vendes aisladas en lado izquierdo de filametho. Trieamas de Nastoc commune en el lado derecho. Fig6, 10-11. Cultivo agarizado en medio $\mathrm{N} 8$ de $\mathbf{S}$, crispem de 5 meses. Fis. 10 Ramifucaciones profusas de filamentos. Fie 11. Filamentos con frecuentes beterocistos y formación de hormogeaios. Fig. 12. Filansentos ramificados pardo verdnso oscuras de $\mathbf{S}$. crispeam en cultivo agarizado en medio NB de 6.5 meses. Fg.13. Filamentos ramificados pardo naranjas de S. crispom de cultivo agarizado en niedie NS de 1.5 atos. Escala en Fig io vátida pars Figs 10, 12:13. 

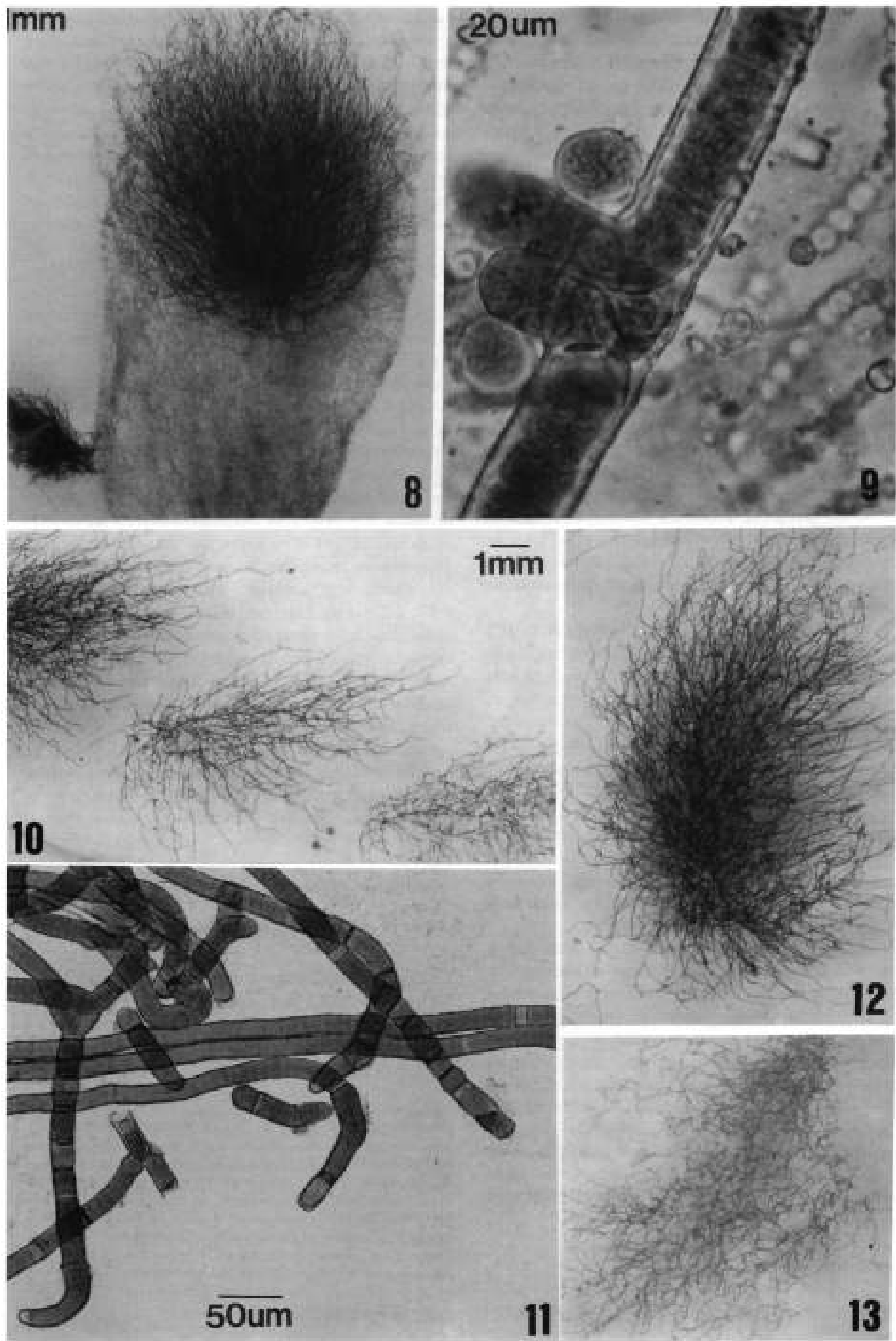
La imbibición de las formas de latencia en medio líquido (Basal Bold) fue evidenciado en el lapso de una semana. Después de la imbibición se re-estableciô la actividad metabólica normal y su crecimiento. La germinación de hormocistos, ligeramente atenuados en sus extremos y variados en longitud implicó cambios morfológicos como incremento en sus dimensiones, alcanzando de 18 a $24 \mu \mathrm{m}$ de diàmetro.

El espesor del estuche estratificado, pardo amariliento o pardo dorado alcanzó hasta $12 \mu \mathrm{m}$. La reactivación diferencial de las células de los talos filamentosos se reconoció por las variaciones en las dimensiones y tonalidades celulares. Los filamentos desecados en los hormocistos con celulas coniraicas y pálidas alcanzaron de 6.4 a 10.7 um de diámetro mientras que las células rípidamente reactivadas y turgentes se presentaron arul verdosas, altanzando de 12.8 a $14 \mu \mathrm{m}$ de diámetro. La emerpencia de las células vegetativas en los extremos de los hormocistos luego del fenómeno de adelgazamiento y disolución de la pared eelular fue evidente con las células apicales intensamente azul verdosas. De esta forma la proliferación celular ejerció presión en los extremos de lass paredes para la emergencia de los filamentos reactivados. Las condiciones favorables de los cultivos para el crecimiento vegetativo también favorecieron la germinación de los hormocistos donde las eélulas experimentan condiciones adecuadas para la fotosintesis y reproducción por fisión.

Los cultivos estacionanos en medio Basal Bold de 2 meses demostraron inicialmente el crecimiento rápido de M. vaginatus. Los filamentos de esta especie formaron una nata fina o película gelatinosa tubular (pox el tipo de recipiente; tubo), pardo verdusca clara o pardo clara, sobre la cual desarrollaron en la parte superior filamentos azul verdosos de S. crispum. Estos filamentos orientados hacia Ia luz adquirieron el aspecto de talos fasciculiformes con frecuentes ramificaciones y alcanzaron de 19 a $20 \mu \mathrm{m}$ de diámetro. Las células intensamente verdosas presentaron un estuche más bien delgado e incoloro, de hasta $4.3 \mu \mathrm{m}$ de espesor (Figs 8-9). Además colonias irregulares de N. commune se presentaron en la parte inferior o hase de la misma nata de $\mathbf{M}$. vaginatus - $\mathbf{S}$, crispum.

Los talos de S. crispum profusamente ramificados, azul verdosos y pardo verdosos fueron comunes en cultivos en medio agarizado (N8) de 5 meses. Las células no se presentaron uniformes en coloración siendo algunas verde azuladas y otras amarillenta pálidas y con estuche delgado incoloro. Igualmente se reconocieron frecuentes heterocistos (Figs, 10-11). En los mismos cultivos de 6.5 meses, los talos filamentosos ramificados de S. crispum se presentaron más intensamente coloreados de pardo olivo o parduscos (Fig. 12). Por el contrario, los filamentos de cultivos de 1.5 años fueron pardo amarillentos, pardo anaranjados 0 verde palidos aunque fueron vables en varics sectores (Fig. 13).

\section{Microcolens vaginatus}

Los cultivos en medio líquido (Basal Bold) de 2 meses evidenciaron que el crecimiento inicial de $\mathbf{M}$. vaginatus fue más rápido que el de S. crispum. Filamentos aislados y atenuados del talo complejo de $\mathbf{M}$. vaginatus alcanzaron de 7.5 a $8.6 \mu$ n de diámetro. Las celulas discoidales, con grínulos de reserviz, alcanzaron de 5.8 a $6.4 \mu \mathrm{m}$ de diámetro por 2.1 a $3.4 \mu \mathrm{m}$ de longitud. Los filamentos aislados con frecuente formación de hormogonios intercalares o terminales fueron semejantes a los del género Lyngbya por el estuche bien definido e incoloro, de hasta $2.4 \mu \mathrm{m}$ de espesor. Los hormogonios liberados del filamento parental se deslizaron para aislarse y desarrollaron nuevos individuos. Célula apical cónica y capitada, de 2.1 a 2.7 um de diámetro y de 1 a $2 \mu \mathrm{m}$ de longitud. (Figs, 20-21).

En medio agarizado (N8) M. vaginatus creció más rápido por las condiciones semi-sôlidas y húmedas que las otras especies asociadas (S, crispum y N. commune). En cultivos de 5 meses, los filamentos fasciculados y atenuados, pardo verdosos claros o pardo amarillentos, rodeados de un estuche incoloro se distribuyeron más bien paralelos, curvados, agregados (paquetes de filamentos) o aistados (Figs, 14-15). Sin embargo, la habilidad de los filamentos de enrollarse en círculos (status circinatus) por motilidad (deslizamiento) sobre la superficie de agar que evidenció su flexibilidad se presentó cuando la densidad del alga interfirió con su motilidad presentándose competencia espacial intraespecifica (Fig. 16). Células vegetativas de 5.4 a 6.4 um de diámetro por 1.6 a $3.2 \mu \mathrm{m}$ de longitud. Filamentos marcadamente atentiados en forma gradual terminan en célula apiçal capitada, de 2 a $3 \mu \mathrm{m}$ de diämetro por 1.4 a $2.7 \mu \mathrm{m}$ de longitud. La variabilidad en la célula apical se reconoce cuando se forman hormogonios en los ápices de los filamentos que luego son liberados dejando una célula apical convexa recientemente formada (Figs. 17-19).

Los talos filamentosos moribundos de M. vaginatus en las mismas placas anteriores (cultivo en agar) pero de 6.5 meses se tornaron blanquecinos con la gradual desecación del medio de cultiva. Sin embargo, S, crispum especie heterocistada cocxistente con M. vaginatus se mantuvo aún viable por un lapso mayor (1.5 años).

\section{DIScusión}

Los paitones climáticos naturales producen ciclos de sequedad seguidos por periodos de humedad que son importantes para el desartollo y distribución de las plantas en especial en las lomas de nuestra costa desértica. La estructura de las costras desćrticas está relacionada con

Figs 14-21. Mierocelesas vaginatus. Figs. 14-19. Cultiva agarizade ell medio N8 de 5 meses. Figs.14-15: Fascicalos de filamentos pardo dorados y tricomses akslados. Pig.16. Tricomas enrollados en cisculos. Fig.17. Percicin apical de tricomas paralelos atenaados. Fig.18. Formación de hormogonio terminal y deslizamiento ent iricoma aislado. Fig. 19. Variabilidad apical de tricomas parafelos

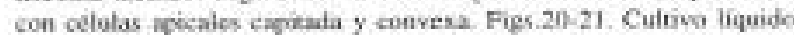
en medio Hasal Bold de inóculo inicial de 2 meses. Fig.20. Triooma atenuado con grimtalos de reserva y célala apical capitada. Fie.21 Furmación de hormogonio con extremos afenuados y capitados en filamento aislato. Escala en Fig.16 valida para Figs. 15-16. Escala en Fiz.18 vilida para Figs.17.21. 

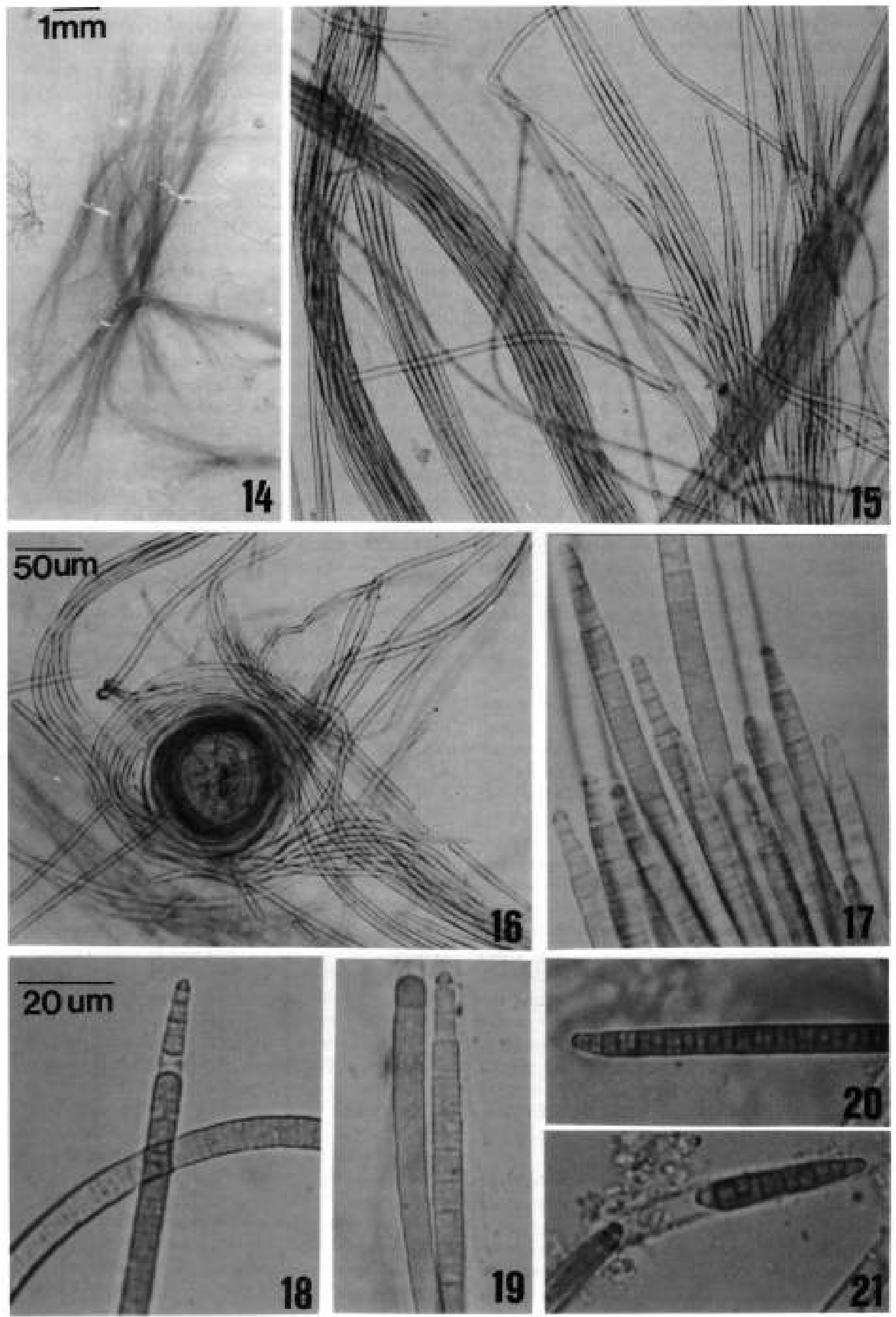
los requerimientos ecológicos y tolerancia de las especies algal-liquénicas consideradas como plantas poiquilohidricas y pioneras en la colonización de ecosistemas desérticos.

La compensación a las fluctuaciones en el abastecimiento de agua en las lomas durante periodos cortos (garias) esta dada por la habilidad de adaptación de los talos criptogámicos al contenido de agua de la humedad del entorno que no siempte se satura pero facilita la colonización algal-liquénica. JEFFRIES et al, (1995) ha indicado que la productividad de las costras desérticas es mayor en condiciones de saturación parcial de agua que durante la saturación completa y BAILEY et al. (1973) deterninó el incremento en la estabilidad del agua en los agregados de particulas del suelo por las al gas asociadas: Nostoc commune, Microcoleus vaginatus (Oscillatoria profilica) y Chlorella vulgaris ( $\mathrm{C}$. pyrenoidosa).

Las cianobacterias que dominan biológicamente las costras desérticas permanecen en estado lafente (inactivo) durante la estación seca (parte de primavera y verano principalmente) en las lomas de Pachacamac y reasumen su actividud sólo cuando el agua es disponible (garias o condensaciones). Las estrategias cianobacteriales de sobrevivencia a la desecación residen en la plasticidad fenotípica de las especies con variabilidad morfológica de sus talos como el gran desarrollo de la pared y estuche circundante. La habilidad de los filamentos de M. vaginatus fue evidenciado por el enrrollamiento en circulos astatus circinatus s creándose microambientes donde pueden permanecer latentes cuando la densidad algal u otro obstáculo (desecación del sustrato) interfiere con se motilidad y crecimiento durante la colonización en las lomas del desierto, $\mathrm{La}$ sobrevivencia a condiciones extremas como la sequedad presente en los desiertos con fluctuaciones climaticas estacionales ha sido reportada para la cianobacteria Nostoc commune (CAMERON 1962. WHITTON 1987). La presencia de filamentos de N. commune redeados con macflago los hace resistentes y favorece su exitosa colonización en periodos desfavorables. En perícodos secos las formas coloniales enteras de N. commune con denso mucilago y filamentos gelatinosos como S. crispum y M. vaginatus se secan periódieamente y sobreviven los talos perennes como lípicas plantas poiquilohidricas que alternan periodos de sequedad y de humedad en las lomas. El desarrollo del estuche lamelado, pigmentado o incoloro en las poblaciones cianobacteriales negruzcas y estables de $\mathbf{S}$. crispum, M. vaginatus y N. commune de las lomas de Pachacamac se avala con el reporte para algas terrestres de curas latitudes (SENGH 1950, SHIELLDS \& DROUET 1962. BELNAP \& GARDNER 1993, FRIEDMANN \& GALUN 1974).

La formación de bormocistos considerados como estructuras especializadas (intermediarios entre hormogonios y acinetos) constituye otra estrategia ecológica de sobrevivencia ciannobacterial. La germinación de hormocistos ba sido reportada para las cianobacterias Scytonema javanicum (Kuetzing) Bornet y Westiella lanosa Fremy (ALLSOPP 1968, FOGG et al, 1973). En las komas de Pachacamac también se presentaron frecuentes hormocistos pigmentados de S. crispum que aunado con la capacidad diazotrófica de esta especic explica su dominancia y perennización en las costras desérticas de Pochacamac.

La presencia de cianobacterias diazotróficas como Scytonema crispum y N, commune en condiciones de humedad incrementan la productjvidad de los ecosistemas desérticos. El incremento en el contenido de nitrógeno y carbono de los suelos por los procesos de fijación de nitrógeno y fotosintesis influencia en el ciclo biogeoquimico de estos nutrientes en las jomas como ha sido observado por EVANS \& EHRLINGER (1993) para otros ecosistemas áridos. La composición especifica de la estructura cianobacterial en las lomas de Pachacamac estuvo dominada por las algas heterocistadas S. crispum y N. commune. El limitado abastecimiento de nutrientes (nitrógeno, agua) en suelos desérticos puede haber creado una presión selectiva que favorece el desarrollo de especies fijadoras de nitrógeno. Esta distribución especifica también se relaciona con la habilidad de sintetizar la scitonemina como pigmento fotoprotector que colorea los estuches de pardo dorado permitiendo su crecimiento bajo intensa iluminación que caracteriza a ecosistemas desérticos calidos (GARCLA-PICHELL \& CASTENHOLZ 1991)

En los cultivos agarizados ( 6.5 meses) se reconoció el fenómeno de exclusión de $\mathbf{M}$. vaginatus y mayor sobrevivencia de S. crispum que puede atribuirse a su capacidad diazotrófica ventajosa en cultivos viejos con limstación de nutrientes. Este fenómeno avala la dominancia de esta última especie en las costas desérticas evaluadas reconociendo la sucesión ecologeiea en las lomas eon la subdominancia de M. vaginatus.

La dinàmica de las costras deserticas que involucra estados de sucesión ecologica en el sur este de Utah. USA, fue demostrada por GARCIA-PICHFI \& BEINAP (1996). Las costras negruzcas evaluadas correspondieron a poblaciones superficiales extensas de Scytonema sp. Nostoc sp y Microcoleus vaginatus. Esta última especie, considerada por los autores como colonizador primario ea desiertos, se distribaye en capas más bien profundas de las costras, estabilizando los suelos y por la motilidad de sus filamentos se protege de la intensa iluminación en ecosistemas desérticos.

La fotosintesis y respiración se reanudaron después de unos minutes de ser humedecidas las costras cianobacteriales y fueron evidenciados por los microambientes formados (GARCIA.PICHEL \& BEL.NAP 1996). Estos eventos también se presentaron durante la reactivación de los talos cianobacteriales desceados en los medios de cultivo y aceleraron la germinación de los hormocistos y posterior proliferación de los filamentos.

El potencial de crecimiento de las comunidades crustáceas algal-liquénicas también está siendo afectado por la destrucción de las costras por pisadas de rebaños de ganado y actividades antropogénicas, que impactin 
negativamente la cohesión y cobertura de las costras. La compactación del suelo y reducción en la disponibilidad de agua alteran los procesos de interacción suelo-planta que repercuten en el patrón sucesional (xerosere) de las lomas. Esta alteración aunada con la fertifización orgánica de los suelos por el ganado favorece la heterogencidad temporal y espacial de los recursos terrestres y constituyc un factor que influencia en la estructura y distribución de las comunidades deserticas de las lomas.
El proceso de desertificación de las tierras áridas y semiáridas causa cambios en los ciclos biogeoquímicos que acarrean perdida de la capacidad productiva de la biósfera y ponen en peligro la recuperación potencial de las costras algal-liquénicas dañadas. Considerando que la pérdida de los suelos desérticos por la erosión es significativa, es preciso la preservación de la integridad de las costras deserticas de importancia en la formación. estabilización y fertilidad de los suelos en nuestra costa.

\section{REFERENCIAS BIBLIOGRÁFICAS}

ALLSOPP, A. 1968. Germination of hermocysts of Scytonema javanieum and the function of blue-green algal heterocysts. Nature. London 220.810.

ANAGNOSTIDIS, K. \& J. KOMAREK. 1988. Modern approxach to the classification system of Cyanophytes 3. Oscillatcriales. Arch. Hydrobiol. Suppl. $80(1-4): 327-472$. (Algological Studies 50-53). Stuttgart.

ANDERSON, D.C. \& S, R. RUSHFORTH. 1976. The crypsogamic flora of desert soil crusts in southers Utah. Nova Hedwigia 28: 69!-729.

BAIL.EY, D. A.P.MAZURAK \& J.R. ROSOWSKL 1973 Ageregation of soil particles by algae. J. Phycol. 9(1):99. 101.

BELNAP, J. 1990. Microbiotic crusts: their role in past and present ecosystems. Park Science. 10(3):3-4.

BEL.NAP, J. \& J. S. GARDNER. 1993. Sol microstructure in the soils of the Colorado Plateas: the role of the cyanobacterium Microcoleus vaginatus. Great Basin Natu. ralist. 53: $40-47$.

BISCHOFF, H.W. \& H.C. BOLD. 1963. Phycological Studies IV. Some algae from Enchanted Rock and related algal species. Univ, of Texas Publ. N"6318.95p.

CAMERON, RE. 1962. Species of Nastec Vucher ecurring in the Sonoran Desert in Arizona. Trans. Am Micrnos, Soc. $81: 379-384$.

CAMPBELL.S.E. 1979, Soil stabilization by a prokaryote desert crust: implications for Precambrian land biota. Origins of life, 9-335-348.

FOGG, G.E., W.D. STEWART, P. FAY \& A.E. WAL.SBY 1973. The blue-green algae. Academic Press. Loodon. $459 \mathrm{p}$.
ROREST, H.\& C.R. WESTON. 1966. Blue-green algae from the Atacama desert of Northern Chile. J. Phycol. 2; 163. 364.

FRIEDMANN, E.I. \& M. GALUN, 1974. Desert algae, lichens and fungi. In Desert Biology. G.W. Brown (ed.) 165-212 p. Academic Press, New York York.

FRIEDMANN, E.I. 1980. Endolithic microbial life in hot and cold deserts. Origins of life. 10:223-235.

GARCIA - PICHEl, F. \& J, BEL,NAP 1996 . Microenvironments and microscale productivity of cyanobacterial desert crusts. J. Ptiycol. 32:774-782.

GARCIA-PICHE1.F.\& R.W.CASTENHOLZ 1991. Characterization and biological implications of scytonemin, a cyanobacterial sheath pigment. J. Phycol, 27: 395-409.

GETTLER, L. 1932. Cyanophyceae In Rabenhorst's Kryptogamenflora von Deutschland, Osterreich und det Schweiz-Akad. Verlagsges, Leipzig 14:1-1196.

JEFFIES, D.L.,S.O.LINK \& J.M. KLOPATEK. 1993.CO fluses of crytogamic crusts. 2. Response to dehydration. New Phyiol. 125: 391-396

KOMAREK.J. \& K. ANAGNOSTIDIS, 1989. Modem approwach to the classification system of Cyanophytes. 4Nostocales. Arch. Hydrobiol. Supl. 82.83: 247-345. Algological Studies 56. Stuttgart.

METTING, B. 1981. The systematies and ecology of soil algac. Botanical Review, 47:195-312.

RUNDEL, P.W. M.O. DILLON, B. PALMA, H.A. MOONEY.SI GULMON\& J.R. EHLERINGER 1991. The phyogeography and ecology of the coastal Alacama and Peruvian deserts. Aliso. 13(1): 1-49. 
SHIELDS, L.M. \& F, DROUET. 1962. Distribution of terrestrial alga within the Nevade test site. Am. J. Bot. 49: $547-554$.

SHIFL.DS. L.M. \& L.W.DURELL. 1964. Algae in relation to soil fertility. Botanical Review 30: 93-128.

SHIFL.DS, L.M., C.MITCHEL \& F. DROUET. 1957. AIga and lichen stabilized surface crusts as soil nitrogen sources. Am. J. Bot, 44; 489-498.

SINGH, R.N. 1950. Reclamation of "Usar" lands in India through biue-green algac. Nature. London. 165: 325-326.
STARKS, T.L,L.E SHUBERT \& PR. TRANNOR 1981. Ecology of soil algae: a review. Phycologia. 20(1): 65-80.

WHITTON, B.A. 1987. Survival and dormancy of bluegreen algae. In Survival and dormancy of microorganisms. Y. Henis (od.) 109-167 p. John Wiley \& Sons. New York.

ZUNICiA, R. 1980, Algas del suelo de Lima y alrodedores. Rev. Per Biol. 2(1):3-7. 